\title{
Submitted Abstracts from Summer School Participants
}

\section{Public Health
Genomics \\ Public Health
Genomics}

Published online: September 14, 2018

\section{Revealing Discrepancies in Guidelines for Genome- Guided Therapeutic Interventions Among Different Research Consortia and Regulatory Bodies \\ S. Koutsilieria, ${ }^{\mathrm{a}, \text {, F. Tzioufa }}{ }^{\mathrm{a}}$, G.P. Patrinos ${ }^{\mathrm{a}, \mathrm{b}}$}

aDepartment of Pharmacy, School of Health Sciences, University of Patras, Patras, Greece; ${ }^{b}$ Department of Pathology, College of Medicine and Health Sciences, United Arab Emirates University, Al Ain, UAE; * Recipient of an Onassis Foundation scholarship

Objectives and Study: Pharmacogenomics aims to rationalize drug use. To date, over 150 drugs have been approved by the US Food and Drug Administration and the European Medicines Agency bearing pharmacogenomics information in their labels. However, no comprehensive lists exist online for clinicians to retrieve the information related to the respective pharmacogenomic biomarkers.

Methods: To create such a comprehensive resource, we have extracted and curated information from the published literature and online resources. Herein, we selected only those pharmacogenomic biomarkers having strong evidence for their clinical utility. Our repository was further enriched with the existing information documented in the major regulatory bodies, such as the Pharmacogenomics Working Parties of the US Food and Drug Administration and the European Medicines Agency.

Results: Our literature mining effort resulted in a total of 370 records, involving correlations between 226 drugs and 95 genes, implicated in drug treatment modalities. From these recommendations, 103 relate to drug toxicity (e.g. adverse drug reactions), 123 are associated with lack of drug efficacy, 45 correspond to both aforementioned cases, while 23 are for information only. Similarities and, most importantly, discrepancies between the regulatory bodies in question were highlighted.

Conclusion: Triangulating between drugs, genes and pharmacogenomic biomarkers represents our effort to transform this repository into a comprehensive and dynamically curated online database catalyzing the application of clinical pharmacogenomics. This online tool will stand as the first example of an one-stop solution to assess in real-time the implication of genomic biomarkers in drug response, leading to treatment individualization.

\section{Dissimilar Integrated Developmental Culturomics as a Multi-Task Regulative Hub for Microbiomic Applications \\ M.E. Kambouris a , A. Stathoulias a , S. Vassilakis, Y. Manoussopoulos ${ }^{\mathrm{b}}$, G.P. Patrinos ${ }^{\mathrm{a}}$ \\ aUniversity of Patras School of Health Sciences, Department of Pharmacy, Laboratory of Pharmacogenomics and Individualized Therapy, Patras, Greece, bPlant Protection Division of Patras, Institute of Industrial and Forage Plants, Patras, Greece}

Culturomics introduced a high throughput format in conventional microbiology mainly by multiplying the number of substrates in an analysis and by introducing automation. The original approach allowed much higher information context than more primitive phenomic formats (a rather limited combination of binary culturing and assimilation tests in uniform wells): continuous growth monitoring allows plotting of growth curves and spotting of phenotypic variations, especially in morphology, which increase the discrimination power of the technique. Thus, it emerges promising for identifying rare, fastidious and hitherto uncultivable pathogens in clinical samples. The exceptionality of Culturomics though, lies in integrating novel modalities into the usual regulatory environment of a culture (dissimilar integration), to achieve better manipulation of microbiota. Different forms of direct energy transfer might achieve, with lower environmental burden, comparable results to (bio)chemicals in both inducing and suppressing growth-dependent functions, like total growth, exponential growth, induction of secondary metabolism, inhibition of sporulation and/or toxin production. Complex pressure waves, such as music, which affect plants, have yet to be tested with microbiota. On the other hand, accurately controlled modalities depend on electrical energy due to technology level and know-how and conductive applications are better targeted and robust. Thus, microbiota treated with basic formats from medical grade, portable device indicate room for manipulating the kinetic parameters of growth, if not the dynamic as well. Detailed such schemes would be invaluable to improve cost-effectiveness in production of, biomass and cosmetic and therapeutic secondary metabolites. Thus, enhanced production of biopharmaceuticals can be expected, with direct implications in rare regulatory, neoplastic and infectious diseases, as development is easier than of conventional pharmaceuticals. Moreover, manipulation of bioremediation, biodegradation and infection parameters, especially in rare diseases, might alter the kinetics towards more favorable results both by up- or down-regulating either the involved microbiota or any antagonistic/synergistic agents. 
Advancing Global Precision Medicine: An Overview of
Genomic Testing and Counselling Services in Malaysia

A. Balasopoulou', F.-M. Mooy 'b D.J. Baker', C. Mitropoulou', E. Skoufas ${ }^{\mathrm{a}}$, A. Bulgiba ${ }^{\mathrm{b}}$, T. Katsila ${ }^{\mathrm{a}}$, G.P.Patrinos ${ }^{\mathrm{a}, \mathrm{d}}$

aDepartment of Pharmacy, School of Health Sciences, University of Patras, Patras, Greece; ${ }^{b}$ Department of Social and Preventive Medicine, Faculty of Medicine, Julius Centre University of Malaya, Kuala Lumpur, Malaysia; 'The Golden Helix Foundation, London, United Kingdom; ' ${ }^{2}$ Department of Pathology, College of Medicine and Health Sciences, United Arab Emirates University, Al Ain, United Arab Emirates

Objectives and Study: Precision medicine, genomic and diagnostic services are no longer limited to developed countries. This broadening in geography of biomarker applications and omics diagnostics also demands empirical study of implementation, diagnostic testing, and counselling practices in the field. For example, the Malaysian population has large ethnic diversity and high prevalence of genetic disorders such as hemoglobinopathies and metabolic disorders. Increased morbidity and mortality from such diseases have a direct impact on society and health system sustainability and for this, decision-making becomes of outmost importance. We report here on our findings on the landscape of genomic testing and genetic counselling services in Malaysia.

Methods: We first defined the framework of all Malaysian stakeholders that offer genomics services and next, we identified the related information gaps, as depicted through the service providers' online websites.

Results: Our research framework revealed that there is a very diverse spectrum of genomics services in Malaysia, in which wetand dry- laboratory services integrate. Moreover, we identify the current gaps and possible remedies to improve the quality of genomic and predictive analytics, not to mention considerations to ensure robust ethics and responsible innovation. To our knowledge, this is the first such study to be performed for a Southeast Asian country.

Conclusion: Our genomic medicine services mapping strategy presented in this study may serve as a model for field assessment at regional, national, and international levels as precision medicine is expanding globally and new governance challenges and opportunities continue to emerge for smart implementation science.

\section{Triangulating Human Microbiome with Genes and Drugs for Clinical Pharmacogenomics: Where Do We Stand and How to Move Forward? \\ A. Balasopoulou ${ }^{\mathrm{a}}$, I. Tsagaraki ${ }^{\mathrm{a}}$, G.P. Patrinos ${ }^{\mathrm{a}, \mathrm{b}}$, T. Katsila ${ }^{\mathrm{a}}$}

${ }^{a}$ Department of Pharmacy, School of Health Sciences, University of Patras, Patras, Greece; bepartment of Pathology, College of Medicine and Health Sciences, United Arab Emirates University

Objectives and Study: The diversity and interactions of the microbial ecosystem in humans become of prime interest when clinical pharmacogenomics is to be implemented. Despite the advent of technology and the extensive use of next-generation sequencing, information still needs to be translated in knowledge.
Pharmacomicrobiomics datasets, which aim to address the interplay of drugs with microbial species and genetics and their effects on drugs' pharmacodynamics and pharmacokinetics, remain multi-faceted and voluminous.

Methods: Herein, we mined and systematically reviewed literature data (as of 2018) to map human microbiome-drug-genes networks. To our knowledge, this is the first time such a thorough strategy has been employed to (i) identify missing information, data complexity and scarcity, (ii) focus on contextual data reliability and curation, (iii) address limitations and challenges, (iv) triangulate data in a comprehensive format.

Results: Our findings, exclusively based on the datasets that survived our inclusion/exclusion criteria, reveal that prebiotics, host-microbe interactions and genetics are the key players, when drug efficacy and/or toxicity are considered.

Conclusion: Such an approach, which facilitated the in-depth exploration and mapping of drug-genes-microbiome networks, may serve as a data storehouse for a user-friendly query system, which will be ideally updated at pre-defined time-points and allow the dynamic data input and curation via microattribution.

\section{Development of an Integrated Health IT Platform for a Precision Medicine Setting}

C. DalleFratte ${ }^{\mathrm{a}}$, R. Roncato ${ }^{\mathrm{a}}$, L. Dal Cin a , F. Comello ${ }^{\mathrm{a}}$, A. Bignucolo,

L. Giollo ${ }^{\mathrm{b}}$, S. D'Errico ${ }^{\mathrm{b}}$, L. Emili', V. Carbone ${ }^{\mathrm{c}}$, M. Guardascione ${ }^{\mathrm{a}}$,

E. De Mattia a , G. Toffolia , E. Cecchin ${ }^{\mathrm{a}}$

aExperimental and Clinical Pharmacology Unit CRO-

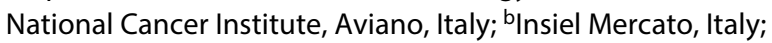

'Promeditech, Italy

Background: Nowadays, clinical validity of many pharmacogenetic markers is undoubted and pharmacogenetics (PGx) studies are carried out by authoritative scientific consortia as the Dutch Pharmacogenetic Working Group (DPWG) and the Clinical Pharmacogenomics Implementation Consortium (CPIC), which lead the development and implementation of pharmacogenetic guidelines. However, the clinical application of these studies in the real life still remains challenging. In fact, the lack of clinical decision-supporting tools that help implementing PGx are highly sought. The integration of the pharmacogenetic data in the electronic clinical records and the development of a decision-support system could be helpful when prescribing the most efficacious and cost-effective pharmacological therapy available. This is particularly relevant in oncology, where the therapeutic drug indexes are narrow and the cost of therapies is high.

Aim: The aim of the project is to radically innovate the prescriptive process of oncological medications by providing physicians with an IT infrastructure (clinical decision-supporting tool) that automatically manage the patients' molecular data, which will be then translated into specific prescriptive indications.

Methods: The most up-dated literature data will be mined to extract a list of gene-drug interactions, based on the most recently published guidelines, to provide a list of the most relevant genetic information that has to be included in the patients' clinical health record, together with the corresponding pharmacogenetic guidelines. 
Results: Publicly available guidelines were reviewed and compared to reach a consensus in terms of the investigated gene-drug pairs, their different level and classification of evidence, which are rated through a scoring system, the recommended therapeutic strategy and the clinical impact of pre-emptive PGx test. Information on the strength of the recommendation (classification of recommendation, level of evidence and clinical impact) were considered necessary to be integrated in the prescribing system along with the therapeutic recommendation. A prototype of a decisionsupport system is being developed through the partnership with two high-tech companies in Italy, which are actively working on the healthcare system computerization.

Conclusions: Scientific and clinical expertise in the oncologic pharmacogenomic field were put together, thanks to the coordinated efforts of an established partnership. The pre-emptive pharmacogenomic approach in the clinical practice in Italy was implemented and demonstrated its benefit in both patients' clinical outcome and quality of life, with an economic advantage for the healthcare system.

\section{Exploring the Gut-Thyroid Axis in Paediatric Coeliac Disease Patients of Hellenic Origin Reveals Selected Genomic Variants in CTLA4, BACH2, and IL23R Genes That May Account for Overlapping Susceptibility Between Graves' Disease and Paediatric Coeliac Disease}

A. Balasopoulou a , D. Kampolisa, M. Krinib , K. Spanouc, A. Johnd,

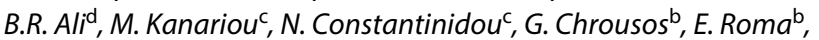
G.P. Patrinos ${ }^{\mathrm{a}, \mathrm{d}}$, T. Katsila ${ }^{\mathrm{a}}$

aDepartment of Pharmacy, School of Health Sciences, University of Patras, Patras, Greece, ${ }^{\text {b}}$ First Department of Pediatrics, University of Athens Medical School, Athens, Greece, 'Department of Immunology and Histocompatibility, "Aghia Sophia" Children's Hospital, Athens, Greece; ${ }^{\text {dDepartment of }}$ Pathology, United Arab Emirates University, College of Medicine and Health Sciences, Al-Ain, UAE

Objectives and Study: Differential diagnosis and theranostics of a series of autoimmune inflammatory disorders remain challenging as we still need to dissect the molecular determinants and cross-talk in the cell signaling of the gut-thyroid axis. Several genetic, epidemiological, clinical, serological, and pathophysiological data indicate that coeliac disease is associated with autoimmune thyroid disorders and in particular, Graves' disease. Today, no clear nomogram is effective to allow for optimum disease management and patient stratification. Herein, we explore the role of selected genomic variants for overlapping susceptibility between Graves' disease and paediatric coeliac disease aiming for an immunogenetic model towards the identification of coeliac disease patients with an increased risk of developing Graves' disease.

Methods: Extensive data mining, pathway analysis and literature review resulted in the selection of CTLA4, BACH2 and IL23R variants. For data validation, coeliac paediatric patients of Hellenic origin $(n=109)$ and their ethnically matched counterparts $(\mathrm{n}=111)$ were genotyped by PCR and Sanger sequencing. HardyWeinberg equilibrium was determined by Pearson's goodnessof-fit chi-square, log-likelihood ratio chi-square and Exact tests.
Genotype and allele frequencies were evaluated by the Fisher's Exact test. A two-tailed p-value of $<0.05$ was considered statistically significant. The $\mathrm{R}$ project for statistical computing ( $\mathrm{R}$ i386 3.2.1) was used.

Results: Selected CTLA4, BACH2 and IL23R variants may account for the overlapping susceptibility between Graves' disease and paediatric coeliac disease in patients of Hellenic origin.

Conclusion: CTLA4, BACH2 and IL23R variants may serve as the building block of a nomogram to optimize Graves' and Coeliac disease management and patient stratification.

\section{Application of Next-Generation Sequencing Technology and Establishment of Biobanks K. Klaassen, M. Stojiljkovic, N. Tosic, B. Zukic, S. Pavlovic}

Institute of Molecular Genetics and Genetic Engineering, University of Belgrade, Belgrade, Serbia

Institute of Molecular Genetics and Genetic Engineering has become widely recognized as an expert centre for rare diseases (RD). It is the first institution is Serbia that applied NGS methodology in research and diagnostics of RD. We are also the institution with RD biobank collections containing DNA, RNA, mononuclear cells and tissue samples from over 2000 of patients affected with 50 different diseases.

An accurate diagnosis was provided to over than $100 \mathrm{RD}$ patients who were undiagnosed for years. We used Clinical-Exome Sequencing TruSightOne Gene Panel (4813 clinicaly-relevant genes), Illumina MiSeq instrument and Illumina VariantStudio. For monogenic diseases, filtration and prioritization of variants were performed according to "in-house" pipeline, using virtual gene panels. Variants were analyzed by various in silico softwares and classified according to ACMG guidelines. Variants selected by these criteria were confirmed by conventional Sanger sequencing and parents' samples were analyzed whenever available.

Furthermore, novel variants in DNAI1, MUT, $P A H, P C C B$, SLC37A4, SPAG16 and SPAG17 genes were functionally characterized in adequate in vitro systems such as immortalized patients' fibroblasts or CRISPR /Cas9 edited commercial cell lines.

Clinical-exome sequencing enabled diagnosis of more than 50 different diagnosis (hematological, metabolic, endocrinological, pulmonary, immunological, orthopedic, dermatological, ophthalmological, cardiological, epileptic encephalopathies etc.). It was particularly important for genetically heterogeneous diseases, such as glycogen storage diseases, branched-chain organic acidurias, primary ciliary dyskinesia, MODY or mitochondriopathies. Moreover, different diseases with overlapping clinical manifestations were accurately diagnosed.

Also, we used TruSeq-Amplicon Cancer Panel to analyse different childhood and adult rare hematological malignancies. Besides studying diagnostic and prognostic malignancy markers, we designed "in-house" virtual pharamocogenomic panel, and performed association studies of pharmacogenomic markers and the course and outcome of rare hematological malignancies, resulting in recommendations for therapeutic modalities in accordance with genomic profile of the patient.

This work has been funded by MESTD, Republic of Serbia (III41004). 


\section{A Functional Genomics Approach Towards PRES (Posterior Reversible Encephalopathy Syndrome) and TMA (Thrombotic Microangiopathy) Management Upon Cyclosporine-A/Tacrolimus Administration \\ C. Chalikiopoulou a, A. Panagiotara a , A. Bitsakos , M-T. Matsoukas ${ }^{\text {a }}$ C. Politopoulou a , C. Kehagia a, E. Gavriilakib , M. Liga ${ }^{c}$, A. John ${ }^{\text {, }}$ B.R. Ali ${ }^{\mathrm{d}}$, A. Anagnostopoulos ${ }^{\mathrm{b}}$, A. Spyridonidis ${ }^{\mathrm{c}}$, G.P.Patrinos ${ }^{\mathrm{a}, \mathrm{d}}$, T. Katsila ${ }^{\mathrm{a}}$ \\ aDepartment of Pharmacy, School of Health Sciences, University of Patras, Patras, Greece, ${ }^{b}$ George Papanicolaou Hospital Hematology Department-BMT Unit; ${ }^{C H e m a t o l o g y ~ D i v i s i o n, ~ B M T ~}$ Unit, University Hospital of Patras, Patras, Greece; ${ }^{\mathrm{d}}$ Department of Pathology, United Arab Emirates University, College of Medicine and Health Sciences, Al-Ain, UAE}

Objectives and Study: Optimum patient stratification becomes vital when fatal toxicities are considered. Herein, we hypothesize that $A B C B 1$ genomic variants correlate to cyclosporine- $\mathrm{A}$ intracellular levels and hence, neurotoxicity and endothelial events.

Methods: Patients who underwent allogeneic HSCT (consent as per transplant center for performing genomic analyses in stored material) and were diagnosed with cyclosporine (or tacrolimus) related Posterior Reversible Encephalopathy Syndrome $(n=3)$ or thrombotic microangiopathy $(\mathrm{n}=15)$ (definitive diagnosis/ severe cases preferred) were retrospectively considered for this study. Data (age at transplant, sex, diagnosis, day of diagnosis of PRES or TMA after transplant, cyclosporine-A/ tacrolimus levels at diagnosis) were collected anonymously. DNA was isolated from donor and from patient pre-transplant blood samples. Genotyping was performed by PCR-RFLP. Method/data validation was employed by Sanger sequencing. In silico and functional analyses were performed via a series of chemo- and bio-informatics tools.

Results: We report that $A B C B 1$ genomic variants correlate with cyclosporine (or tacrolimus) related PRES and TMA and thus, could be operative in the occurrence of these complications. In silico and functional analyses provide a rationale for such a genotype-to-phenotype association.

Conclusion: This is the first time a functional genomics approach reveals that $A B C B 1$ genomic variants correlate with PRES and TMA outcomes in Greek patients who underwent allogeneic HSCT and sheds light on such a genotype-to-phenotype association.

\section{A Multi-Omics Strategy May Reveal the "Actionable Genome" and Empower Risk Assessment of Recurrent Cardiovascular Events}

C. Chalikiopoulou', I. Liopetas ${ }^{\mathrm{a}}$, A. John ${ }^{\mathrm{b}}$, A. Menti ${ }^{\mathrm{c}}$, B.R. Ali ${ }^{\mathrm{b}}$, G. Hahalis', T. Katsila ${ }^{\mathrm{a}}$, G.P.Patrinos ${ }^{\mathrm{a}, \mathrm{b}}$

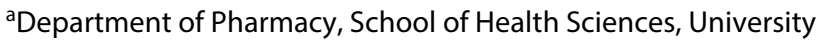

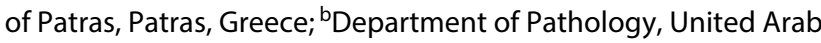
Emirates University, College of Medicine and Health Sciences, Al-Ain, UAE; ' Cardiology Department, School of Medicine, University of Patras, Greece

Objectives and Study: Cardiovascular disease remains a leading cause of death and at the same time, a global public health issue of major socio-economic impact. Optimal patient stratification and decision-making in the clinic demand the in-depth understanding of the (patho)physiology of the cardiovascular system, not only for a cardiovascular event to be successfully dealt with, but also to assess the risk of its recurrence. Herein, we aim to perform a state-of-the-art strategy towards risk assessment and betterinformed patient stratification via multi-omics data integration.

Methods: Following the recruitment of patients with a cardiovascular event (first or a recurrent one) of Hellenic origin, we performed text- and data- mining and used a series of databases and chemoinformatics to explore a panel of genomic variants and their association to cardiovascular events and their recurrence. Such datasets are integrated to miRNAs and proteomics/metabolomics datasets to reveal the so-called "actionable genome" and map inter-individual variability.

Results: Our preliminary datasets indicate that selected NOS3, NOA1, PHACTR1, PCSK9, APOB, MRAS, GUCY1A3, CDKN2B$A S 1, B C A S 3, V E G F$ and SMARCA4 variants may account for the cardiovascular events in question and their recurrence.

Conclusion: Such a multi-omics strategy may serve as the building block of a nomogram to optimize cardiovascular disease management and patient stratification with an emphasis on risk assessment. 
Cost-Effectiveness Analysis of PharmacogenomicsGuided Clopidogrel Treatment in Spanish Patients Undergoing Percutaneous Coronary Intervention

V. Fragoulakis ${ }^{\mathrm{a}}$, M. Bartsakoulia ${ }^{\mathrm{b}}$, X. Diaz ${ }^{\mathrm{c}}$, C. Chalikiopoulou ${ }^{\mathrm{b}}$, C. Kehagiab ${ }^{\text {, J.G. Sánchez-Ramos }}{ }^{\text {d, L.J. Martínez-Sánchez }}{ }^{\mathrm{e}}$, M. Gkotsi ${ }^{\mathrm{b}}$, E. Katrali ${ }^{\mathrm{b}}$, E. Skoufas ${ }^{\mathrm{b}}$, A. Vozikis ${ }^{\mathrm{f}}$, A. Johng ${ }^{\mathrm{g}}$, B.R. Alig, S. Wordsworth h,i, C.L. Dávila-Fajardoc, T. Katsila ${ }^{\mathrm{b}}$, G.P.Patrinos ${ }^{\mathrm{b}, \mathrm{g}}$, C. Mitropoulou

aThe Golden Helix Foundation, London, UK; ' University of Patras School of Health Sciences, Department of Pharmacy, Patras, Greece; ' University of Granada, San Cecilio University Hospital, Granada, Spain, dCardiología, hospital Universitario San Cecilio/hospital Campus de la Salud, Institute for biomedical research, ibs.GRANADA, Granada, Spain, 'Genomics Unit, Centre for Genomics and Oncological Research (GENYO), University of Granada, Health Sciences Technology Park, Granada, Spain; fUniversity of Piraeus, Economics Department, Piraeus, Greece; 9United Arab Emirates University, College of Medicine and Health Sciences, Department of Pathology, Al-Ain, UAE; hUniversity of Oxford, Health Economics Research Centre, Nuffield Department of Population Health, Oxford, UK; i Oxford National Institute for Health Biomedical Research Centre

Objectives and Study: According to World Health Organization, cardiovascular disease is a leading cause of death, worldwide. The most common type of cardiovascular disease is Coronary Artery Disease and the majority of patients diagnosed with it undergo Percutaneous Coronary Intervention. The main type of antiplatelet treatment is clopidogrel. CYP2C19 genomic variants are associated with variable enzyme activities affecting clopidogrel metabolism, resulting in reduced or increased enzymatic function. A cost-effectiveness analysis was conducted to compare a pharmacogenomics versus a non pharmacogenomics-guided clopidogrel treatment for coronary artery syndrome patients undergoing PCI in the Spanish healthcare setting.

Methods: 549 patients diagnosed with coronary artery disease followed by PCI and clopidogrel treatment were recruited. Patients were classified into (i) the retrospective group, treated with clopidogrel based on the clinical routine practice and the prospective group, being genotyped for $C Y P 2 C 19$ before treatment and those carrying more than one $C Y P 2 C 19$ variant alleles given prasugrel treatment. We collected data on established clinical and health outcome measures. Our primary outcome measure for the costeffectiveness analysis was Quality Adjusted Life Years.

Results: Our results predict a survival of 0.9446 QALYs in the pharmacogenomics -arm and 0.9379 QALYs in the non- pharmacogenomics one within an one-year horizon. The cumulative costs per patient were $€ 2,971$ and $€ 3,205$ for the Prospective and Retrospective groups, respectively. The incremental cost-effectiveness ratio was negative indicating that the PGx was a dominant option.

Conclusions: The pharmacogenomics-guided clopidogrel treatment strategy may represent a cost-effective choice compared with non-pharmacogenomics-guided strategy for patients undergoing PCI.

\section{A Molecular Map for Glioblastoma Multiforme Patients via Multi-Omics Datasets \\ T. Katsila ${ }^{\mathrm{a}, \mathrm{b}}$, M. Pittaka ${ }^{\mathrm{a}}$, A. Panagiotara ${ }^{\mathrm{b}}$, V. Zolota ${ }^{\mathrm{c}}$, \\ G.P. Patrinos ${ }^{\mathrm{b}, \mathrm{d}}$, D. Kardamakis ${ }^{\mathrm{a}}$}

aDepartment of Radiation Oncology, University of Patras

Medical School, Patras, Greece; 'bepartment of Pharmacy, School of Health Sciences, University of Patras, Patras, Greece; 'Department of Pathology, University of Patras Medical School, Patras, Greece; d Department of Pathology, College of Medicine and Health Sciences, United Arab Emirates University, Al Ain, UAE

Objectives and Study: Primary tumors of the central nervous system are characterized by molecular heterogeneity, which is expressed by different clinical phenotypes. The study of the biology of these tumors, and in particular of the multiple glioblastoma (PG), continues to be a challenge in modern oncology, considering the poor prognosis of the disease. Herein, we aim to integrate miRNA expression data and metabotypes with the overall survival of glioblastoma multiforme patients.

Methods: We focused on miR-20A, miR-10A and miR21 which relate to glioblastoma multiforme biology and determined the metabolomic phenotypes for each patient. Molecular (RT-qP$\mathrm{CR}$ ) and analytical (liquid chromatography mass spectrometry) techniques were applied and a total of 123 serum samples and 9 pathological preparations were analyzed in a sample of 22 patients with histologically diagnosed glioblastoma multiforme.

Results: Our findings report i) high expression levels for miR20a, miR-10a and miR21 in all histopathological preparations (>2fold) and ii) metabotypes were associated with the heterogeneity of the disease and overall survival. Anti-apoptotic metabotypes and metabotypes that promote cell proliferation were associated with reduced overall survival.

Conclusion: Our strategy may serve as a paradigm and shed light on glioblastoma multiforme genotype-to-phenotype associations.

\section{Spectrum and Clinical Manifestations of Mutations in PIEZO1}

K. Maciak ${ }^{\mathrm{a}}$, A. Adamowicz-Salach ${ }^{\mathrm{b}}$, A. Siwicka ${ }^{\mathrm{b}}$, T. Urasinskic, B. Burzynska

anstitute of Biochemistry and Biophysics, Polish Academy of Sciences, Warsaw, Poland; 'bepartment of Paediatrics, Haematology, and Oncology, Warsaw Medical University, Poland; ' Department of Pediatrics, Hematology and Oncology, Pomeranian Medical University, Szczecin, Poland

Background: Hereditary xerocytosis (HX) also known as dehydrated hereditary stomatocytosis is the red cell membrane disorder resulting in hemolytic anemia with various presentation and iron overload. Dominantly inherited missense mutations in PIEZO1 gene encoding large homotetrameric mechanosensitive ion channel, located mainly in the highly conserved $\mathrm{COOH}$-terminus, have been identified recently in HX patients. The aim of our study was to elucidate the molecular basis of hereditary xerocytosis in 4 Pol- 
ish patients with suspected HX or undiagnosed congenital hemolytic anemia.

Materials and Methods: Total RNA was isolated from peripheral blood using PAXgene Blood RNA Kit (QIAGEN, Germany) and reversely transcribed. DNA fragments generated by PCR amplification were directly sequenced. Genomic DNA was extracted from peripheral blood using standard methods. The presence of detected mutations on the cDNA level was verified by sequencing using genomic DNA as template. The detected variants were evaluated for potential pathogenicity by MutationTaster, PROVEAN, PolyPhen-2 and M-CAP software.

Results: All probands exhibit mild to moderate compensated hemolytic anemia with the presence of stomatocytes in the blood smear. Direct sequencing of PIEZO1 gene of four patients showed different mutations: (1) c.2005 G>C mutation that results in the substitution p.D669H, (2) c.4697 A>T substitution that predicts aspartic acid to valine at the position p.D1566V, (3) c.2233_2247 deletion CAG that removes one glutamine from the glutamines repeat at position p.745_749,(4) c.5195C > T mutation which results in substitution threonine to methionine at position p.T1732M. Since, Patient 4 showed elevated level of $\mathrm{HbF}$ and $\mathrm{HbA} 2$, we performed sequencing of $\beta$-globin gene. Sequence analysis demonstrated the presence of deletion 12 nucleotides (GCTCATGGCAAG) involving codons $62,63,64$ and 65 and this results in deletion of $A, H, Q$ and K. Deletion of these residues results in deformation of heme pocket particularly in the distal side, so it may suggest disorder in oxygen - iron complex formation.

Conclusions: Molecular analysis showed four different mutations in PIEZO1 gene, including two novel mutations. In two cases (Patient 2 and 3 ) we observed a mild manifestation of HX. In contrast, Patient 4 with coinherited $\beta$-globin variant, showed more severe clinical course and had a significant history of transfusions. It speaks in favour of the hypothesis that coinherited mutations would cause a more severe phenotype.

\section{Genotype to Phenotype Associations with an Emphasis on the Response and Pharmacokinetics of Tacrolimus in Kidney Transplantation Patients of Hellenic Origin \\ E. Mendrinou ${ }^{\mathrm{a}}$, S. Papageorgiou ${ }^{\mathrm{b}}$, M. Papasotiriou ${ }^{\mathrm{b}}$, \\ E. Papachristos ${ }^{\mathrm{b}}$, K. Kydonopoulou', E. Papadopoulou', S. Gerou', \\ D. Goumenos ${ }^{\mathrm{b}}$, M. Bartsakoulia ${ }^{\mathrm{a}}$, T. Katsila ${ }^{\mathrm{a}}$, G.P.Patrinos ${ }^{\mathrm{a}, \mathrm{d}}$ \\ aDepartment of Pharmacy, School of Health Sciences, University

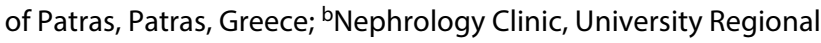 General Hospital of Patras, Greece; 'ANALYSI Diagnostic Laboratories S.A, Thessaloniki, Greece; ${ }^{\mathrm{d}}$ Department of Pathology, College of Medicine and Health Sciences, United Arab Emirates University, UAE}

Objectives and Study: Tacrolimus is an immunosuppressive drug that is widely used as primary therapy following kidney transplantation to prevent acute graft rejection and promote immunological adaptation. Tacrolimus' advantages are offset by its side effects, its narrow therapeutic index as well as its rather unpredictable pharmacokinetics. Herein, we explore genotype-tophenotype associations with an emphasis on the role of $C Y P 3 A 5^{*} 3$ in drug response and pharmacokinetics of tacrolimus in kidney transplantation patients of Hellenic origin

Submitted Abstracts from Summer School Participants
Methods: $C Y P 3 A 5^{*} 3$ genotyping was performed in 92 patients. The dosing regime and blood levels of tacrolimus were recorded and the graft outcome was monitored.

Results: Based on genotyping data, patients were clustered as normal, intermediate and poor metabolizers. CYP $3 A 5 * 3 / * 3$ appear to have a higher $\mathrm{C} / \mathrm{D}$ ratio after first treatment (day 1 ) than CYP $3 A 5^{*} 1 /{ }^{*} 1$ or $* 1 / * 3$ patients $(\mathrm{p}=0.002)$. The $* 3$ frequency in the population study is in agreement $(\sim 91 \%)$ to that reported previously.

Conclusion: Our findings suggest that $C Y P 3 A 5^{*} 3$ genotyping prior to Tacrolimus' treatment may reduce dose adjustments and the time required to achieve the desired drug levels in the blood.

\section{Frequency of CYP2C19 Polymorphism in Ischemic Patients \\ A. Shawky}

Must University, Elobour, Egypt

Human cytochrome P450, particularly CYP2C19 play important role in drug metabolism. The polymorphisms of this enzyme give rise to change in variability in drug excretion rate and function. in this study we determined genotypes of CYP2C19 in the Egyptian population to compare allele frequencies to other ethnic groups. Allelic variants were determined in 110 ischemic Egyptian volunteers by PCR. The results show that $48 \%$ are extensive metabolizers $\left({ }^{*} 1 /{ }^{*} 1\right), 18 \%$ intermediate metabolizers $\left({ }^{*} 1 /{ }^{*} 2,{ }^{*} 1 /{ }^{*} 3\right.$, $\left.{ }^{*} 2 /{ }^{*} 7\right)$ and $34 \%$ are ultra-rapid metabolizers $\left({ }^{*} 1 /{ }^{*} 17,{ }^{*} 17 /{ }^{*} 17\right)$.

\section{Selected Genomic Variants in TNF-Alpha, IL 10, LTA and IRF4 Genes May Account for Overlapping Susceptibility Between Non-Hodgkin Lymphoma and Paediatric Celiac Disease Patients of Hellenic Origin}

A. Panagiotara ${ }^{\mathrm{a}}$, M. Krini ${ }^{\mathrm{b}}$, K. Spanou $^{\mathrm{c}}$, A. John ${ }^{\mathrm{d}}$, B.R. Alid, M. Kanariou', N. Constantinidou', G. Chrousos ${ }^{\mathrm{b}}$, E. Roma $^{\mathrm{b}}$, G.P.Patrinos ${ }^{\mathrm{a}, \mathrm{d}}$, T. Katsila ${ }^{\mathrm{a}}$

aDepartment of Pharmacy, School of Health Sciences, University of Patras, Patras, Greece, ${ }^{b}$ First Department of Pediatrics, University of Athens Medical School, Athens, Greece, 'Department of Immunology and Histocompatibility, "Aghia Sophia" Children's Hospital, Athens, Greece; ${ }^{\text {dDepartment }}$ of Pathology, United Arab Emirates University, College of Medicine and Health Sciences, Al-Ain, UAE

Objectives and Study: Although common variations in genes of the immune system may predispose to chronic inflammatory disease and malignant diseases of the lymphoid system, it is unclear how to identify celiac disease patients with an increased risk of developing a lymphoproliferative disorder. Herein, we explore single nucleotide polymorphisms in selected genes for overlapping susceptibility between non-Hodgkin lymphoma and paediatric celiac disease.

Methods: Extensive data mining, pathway analysis and literature review resulted in the selection of genomic variants in TNF-alpha, IL10, LTA and IRF4 genes. For data validation, paedi- 
atric celiac disease patients of Hellenic origin $(n=109)$ and their healthy ethnically matched counterparts $(n=111)$ were genotyped by allele-specific PCR, PCR-RFLP and Sanger sequencing. Hardy-Weinberg equilibrium was determined using the chisquare and Fisher exact tests. Genotype and allele frequencies were evaluated using the Fisher exact and the Cochran-Armitage trend tests. A two-tailed p-value of $<0.05$ was considered statistically significant. Further data analysis with in silico prediction models was implemented to determine functionality (Variant Effect Predictor, Human Splicing Finder, Gene Promoter Miner, RegulomeDB).

Results: Our results indicate a statistically significant association of selected genomic variants in TNF-alpha ( $\mathrm{p}<0.0000001)$, IL10 $(\mathrm{p}<0.0001)$ and LTA $(\mathrm{p}=0.016)$ with celiac disease patients of Hellenic origin, but not in IRF4 ( $\mathrm{p}=0.729)$ which showed no correlation with the disease, possibly because it is considered a population substructure marker.

Conclusion: Selected genomic variants in TNF-alpha, IL10, LTA and IRF4 genes are candidate genomic markers for overlapping susceptibility between non-Hodgkin lymphoma and paediatric celiac disease patients of Hellenic origin, empowering patient stratification.

\section{Liquid Biopsies in Clinical Practice: Early Detection of Imatinib Resistance Subclones in GIST patients}

C. DalleFratte ${ }^{\mathrm{a}}$, M. Guardascione ${ }^{\mathrm{b}}$, A. Buonadonna ${ }^{\mathrm{b}}$, L. Foltran', R. Loredana a , E. De Mattia ${ }^{\text {a }}$, G. Toffoli ${ }^{a}$, E. Cecchin ${ }^{\text {a }}$

aExperimental and Clinical Pharmacology Unit, CRO-National Cancer Institute, Aviano, Italy; ${ }^{b}$ Medical Oncology Unit, CRONational Cancer Institute, Aviano, Italy; 'Medical Oncology, Azienda per l'Assistenza Sanitaria 5, Pordenone, Italy

Background: Gastrointestinal stromal tumor (GIST) represents the most common soft tissue sarcoma and is frequently characterized by KIT and PDGFRA activating mutations (Singer et al., J Clin Oncol. 2002;20(18):3898-3905; Heinrich et al., Science. 2003;299(5607):708-710). Although the use of tyrosine kinase inhibitors (TKi), such as Imatinib, yielded great improvements in the management of GIST, many patients, who initially responded positively to Imatinib, developed secondary resistance against chemotherapy, thus experiencing tumor progression (Demetri et al., N Engl J Med. 2002;347(7):472-480). Since the onset of secondary mutations in tumor cells represents the leading mechanism of acquired drug resistance, early identification of those resistant subclones could improve the decision making in the management of chemotherapy, thus preventing delays in drug administration and disease progression. With this in mind, the analysis of circulating tumor DNA (ctDNA) in clinical practice might represent a suitable tool for the real-time monitoring of tumor to better tailor the drug's prescription (Crowley et al., Nat Rev Clin Oncol. 2013;10(8):472-484).

Aim: The project aims at assessing the feasibility and applicability of ctDNA sequencing in clinical practice by identifying the somatic mutations that are potentially involved in the development of acquired chemo-resistance. This ambitious goal can be achieved by analyzing ctDNA collected from GIST patients administered with Imatinib.
Methods: Patients administered with Imatinib are being enrolled in a prospective manner and $10 \mathrm{ml}$ of serials blood samples will be collected during the entire course of chemotherapy. Blood will be drawn using PAXgeneccfDNA collection tubes (QIAgen) in order to prevent the white blood cells lysis. Plasma cell free DNA (cfDNA) will be extracted from $4 \mathrm{ml}$ of plasma using QIAampMinEluteccfDNA kit and fragment distribution will be assessed with High Sensitivity TapeStation (Agilent Technologies). ctDNA detection by Next Generation Sequencing (NGS) will be performed on an Illumina platform (MiSeq) with short reads (150 bp) analyzing a targeted panel comprising all exonic and splice site regions (QIAgen) of eleven onco-genes, including cKIT, PDGFRA, BRAF, TP53; and mTOR, involved in the development of Imatinib-acquired resistance. The ultrasensitive variant detection will be achieved using integrated unique molecular indices (UMIs), while the bioinformatic analysis will be automatically performed using smCounter pipeline (Xu et al., BMC Genomics. 2017;3;18(1):5). All somatic mutations will be confirmed using ddPCR (Bio Rad).

Results: Pre-analytical conditions for cfDNA handling were set up including extraction, quantification and quality assessment. Up to date 26 patients were enrolled and 8 samples were investigated. Preliminary data showed that cfDNA plasma concentrations were typically low but extremely variable among patients, ranging from 3 to $101 \mathrm{ng} / \mathrm{ml}$ of plasma. To date a never-described somatic mutation in TP53 was identified in two serial blood samples belonging to the same patient experiencing tumor progression. The presence of such somatic mutation was validated through ddPCR assay in cfDNA and its functional relevance will be assessed by further analysis. A population of 60 patients administered with Imatinib is expected to be enrolled by the end of 2019 .

Conclusions: Tremendous advantages will arise from cfDNA analysis as routine clinical practice to early identify onset of drugresistance. In fact, cfDNA analysis could represent a suitable tool not only to better understand the mechanisms underlying tumor evolution but also to early provide alternative therapeutic strategies for the management of chemotherapy, such as Sunitinib.

\section{Using Computational Methods to Map Multigenic Disease Phenotypes: An ALS Toolbox}

S. Papadaki ${ }^{a}$, R. Siakavaras a , C. Chalikiopoulou a , A. Balasopoulou ${ }^{\text {a }}$, S. Koutsilieri, ${ }^{\mathrm{a},}$, A. John ${ }^{\mathrm{b}}$, B.R. Ali ${ }^{\mathrm{b}}$, T. Katsila ${ }^{\mathrm{a}}$, G.P. Patrinos ${ }^{\mathrm{a}, \mathrm{b}}$

aDepartment of Pharmacy, School of Health Sciences, University of Patras, Patras, Greece; ${ }^{b}$ Department of Pathology, College of

Medicine and Health Sciences, United Arab Emirates University,

Al Ain, UAE; * Recipient of an Onassis Foundation scholarship

Objectives and Study: Precision medicine aims at optimal disease management and patient stratification and, in this perspective, mapping of phenotype-to-genotype association becomes vital. Considering the complexity of multigenic disease phenotypes and inter-individual variability, better-informed decisions need valid datasets. Herein, whole genome datasets were explored to reveal genomic variants of interest.

Methods: Following extensive bio- and chemo-informatics analyses coupled to $\mathrm{R}$ (programming language), we focused on genomic variants that were unique a) per disease phenotype (Behcet's vs. ALS vs. spastic paraparesis), b) in autoimmune disease pheno-
2018 Golden Helix Summer School Rare Genomics 
types of interest (Behcet's plus ALS plus spastic paraparesis), c) in neurodegenerative disease phenotypes of interest (amyotrophic lateral sclerosis plus spastic paraparesis) and/ or metabolic disorders (Behcet's disease). In total, 260,671 genomic variants were assessed and SLC22A24 genomic variants were further validated by PCR and Sanger sequencing in ALS patients of Hellenic origin.

Results: Our findings demonstrate the existence of significant genomic consistency among the disease phenotypes in question, with 12,612 genomic variants being in common. Interestingly, these genomic variants are predominantly found in chromosomes 7, 8 and 11. Moreover, genomic variants unique to ALS and/or spastic paraparesis have been highlighted.

Conclusion: Our wet- and dry-lab approach may serve as a paradigm to reveal the "actionable genome" and empower data reliability in multigenic disease phenotypes.

\section{Genomic Variants in the FTO Gene are Associated with Sporadic Amyotrophic Lateral Sclerosis in Greek Patients}

K. Mitropoulos a , E. Merkouri Papadima ${ }^{b}$, G. Xeromerisiou', A. Balasopoulou ${ }^{\text {, }}$ K. Charalambidou ${ }^{\mathrm{b}}$, V. Galani ${ }^{\mathrm{b}}$, K-V. Zafeiri $^{\mathrm{b}}$, A. John' ${ }^{\mathrm{d}}$, K. Kydonopoulou', E. Papadopoulou' ', A. di Pardo ${ }^{\mathrm{f}}$, F. Akcimen ${ }^{g}$, A. Loizedda h,i, V. Dobričić, l. Novaković,j, , V.S. Kostićj,

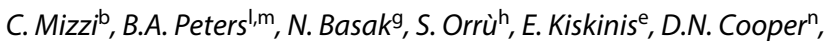
S. Gerou', M. Bartsakoulia' , E.E. Tsermpini ', G.M. Hatzigeorgiou', R. Drmanad, B.R. Alid , T. Katsila ${ }^{\mathrm{b}}$, G.P.Patrinos ${ }^{\mathrm{b}, \mathrm{d}}$

${ }^{a}$ National and Kapodistrian University of Athens School of Medicine, Athens, Greece, b ${ }^{\text {Department }}$ of Pharmacy, University of Patras School of Health Sciences, Patras, Greece, 'Papageorgiou Hospital, Thessaloniki, Greece; dUnited Arab Emirates University, College of Medicine and Health Sciences,

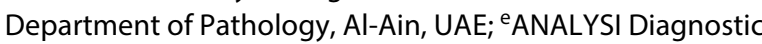
Laboratories S.A., Thessaloniki, Greece; ${ }^{f}$ Northwestern University, Feinberg School of Medicine, Chicago, IL, USA; gBogazici University, Department of Molecular Biology and Genetics, Istanbul, Turkey; hUniversity of Cagliari, Department of Medical Sciences and Public Health, Cagliari, Italy, 'CNR IRGB, Cagliari, Italy; ${ }^{j}$ Institute of Neurology CCS, School of Medicine, University of Belgrade, Belgrade, Serbia, kFaculty of Medicine, University of Belgrade, Institute of Human Genetics, Belgrade, Serbia; 'Complete Genomics Inc, Mountain View, CA, USA; ${ }^{m} B G I$ Shenzhen, Shenzhen, People's Republic of China; ${ }^{\text {nCardiff }}$ University, Institute of Genetic Medicine, Cardiff, UK; ${ }^{\circ}$ School of Medicine, University of Thessaly, Larisa, Greece

Background: Amyotrophic lateral sclerosis is a serious genetic illness, whose complex pathobiology has been associated with a strong genetic component for both familial and sporadic etiology. We adopted a next-generation sequencing approach in Greek patients suffering from sporadic ALS and their healthy counterparts to explore further the genetic basis of sporadic ALS.

Results: Whole-genome sequencing analysis of Greek sALS patients revealed a positive association for FTO and TBC1D1 variants, while linkage disequilibrium analyses suggest a haplotype of FTO gene variants. FTO was found to be highly expressed in motor neurons, while in silico analyses predicted an impact on splicing for the genomic variants in question. Data were validated in Greek, Sardinian and Turkish sporadic ALS patients, where we found lack of association of FTO and TBC1D1 variants with sporadic ALS in patients of Sardinian and Turkish descent, suggesting a founder effect in the Greek population.

Conclusions: To our knowledge, this is the first study denoting a possible association between FTO gene variants and the genetic basis of sporadic ALS.

\section{Correlation of KLF Genomic Variants with Disease Severity and with Hydroxyurea Treatment Efficacy in $\boldsymbol{\beta}$-Hemoglobinopathies Patients}

A. Stratopoulos ${ }^{\text {, A. Aolliopoulou }}{ }^{\text {a }}$, K. Karamperis ${ }^{\text {a }}$, S. Tsekoura ${ }^{\text {a }}$, K. Tziolia a , T. Katsila a , M. Bartsakoulia ${ }^{\mathrm{a}}$, A. Papachatzopoulou ${ }^{\mathrm{b}}$, G.P.Patrinos ${ }^{\mathrm{a}, \mathrm{c}}$

aUniversity of Patras School of Health Sciences, Department of Pharmacy, Patras, Greece, 'baboratory of General Biology, Faculty of Medicine, University of Patras, Greece; ' $D$ Department of Pathology, College of Medicine and Health Sciences, United Arab Emirates University, Al Ain, UAE

$\beta$-Type hemoglobinopathies represent hematological disorders which mainly come up due to genomic variants located to the $H B B$ gene and either result in an imbalance between $\alpha$-and $\beta$-polypeptide chains (quantitative disorders, such as $\beta$-thalassemia) or in the production of different types of hemoglobin (qualitative disorders, such as sickle cell disease-SCD). Reactivation of fetal hemoglobin $(\mathrm{HbF})$ offers various clinical benefits to patients suffering from $\beta$-thalassemia and SCD, because, in $\beta$-thalassemia, HbF production increase compensates for the underproduction of $\beta$-globin chains and, in SCD, it disrupts sickle hemoglobin polymerization. Thus, for the past few years, efforts have been made for the identification of an effective way which would lead to $\mathrm{HbF}$ induction. Multiple chemical HbF inducers have been found, but hydroxyurea (HU) is the only Food and Drug Administration-approved drug which is mostly administered for use in the management of sickle cell disease symptoms.

Kruppel-like family of transcription factors (KLFs) are a set of zinc finger DNA-binding proteins that regulate gene expression and are capable of binding GC-rich sequences. They play crucial role in the development and in erythroid cell differentiation and maturation, as well as in the $\gamma$-to- $\beta$ globin gene switching and in a number of other cellular processes.

In this study, we examine the association of a number of single nucleotide polymorphisms (SNPs) located to KLF3, KLF4, KLF9 and KLF10 genes with the severity of $\beta$-thalassemia, as well as their association with the response of patients to HU treatment. Our findings further underline an association of a SNP located in KLF4 gene with the response to HU treatment and a correlation of a SNP in $K L F 10$ as far as the severity of the disease is concerned. 


\section{Workshop and Continuing Medical Educations for Health Care Professionals}

A. Rahma

Institute of Public Health, College of Medicine and Health

Sciences, United Arab Emirates University, Al-Ain, UAE

Background: Lack of adequate health care professionals' knowledge in Genomics and pharmacogenomics is one of the barriers for adopting Precision medicine:

Aim: in this study we evaluated the outcome of workshop addressing genomic medicine and pharmacogenomics on the knowledge of healthcare providers in the United Arab Emirates.

Methods: A cross-sectional study of healthcare providers and medical students in UAE. We asked the participants in the first Zayed Center workshop in genomics and pharmacogenomics, organized jointly with the 20th Golden Helix Pharmacogenomics Day (February 3rd, 2018, Al-Ain, UAE) to answer a pre- and postconference questionnaire to evaluate the change in their knowledge, resulting from this workshop.

Results: 43 completed questionnaires were included in the analysis of which 19 came from pharmacists, 11 from students and 5 from nurses. Data had been analyzed using Data Analysis and Statistical Software (STATA) in which we used Wicoxon Rank Sum to compare Post score with correlates as well as KruskalWallis equality-of-populations rank test. Despite the small number of respondents, we found that the profession and whether the healthcare providers studied pharmacogenomics or not during their undergraduate studies as the only two statistically significant correlates with improving knowledge of pharmacogenomics and genomics.

Conclusions and Recommendation: Workshops and Continuing Medical Education related to genomic medicine and pharmacogenomics may contribute in bridging the genomics and pharmacogenomics knowledge gap of healthcare providers and that will speed up the adoption of precision medicine.

\section{Preparative DNA Concentration by Epitachophoresis F. Foret, V. Datinska, I. Voracova, P. Gheibi, J. Berka, Y. Astier}

Czech Academy of Sciences, Institute of Analytical Chemistry, Brno, Czech Republic; Roche Sequencing Solutions, Pleasanton, CA, USA

Many clinical and diagnostic applications require high quality nucleic acids for downstream analytical methods such as quantitative PCR, microarrays, and/or next-generation sequencing. A complex matrix, such as blood and other bodily fluids, samples from the scene of crime, fossil samples, etc., very often compromise isolation and purification of nucleic acids.

The most common nucleic acids isolation techniques are based on extraction with inherent limitations, with regard to quantitative results. In recent years, there is an increasing interest in sorbent free alternatives. Here, we report on two instrumental systems for processing of medium to large sample volumes by isotachophoresis and a new mode of discontinuous electrophoresis - epitachophoresis, providing theoretically unlimited concentration factor.
In the first system, we used capillary instrument with large bore ( $0.8 \mathrm{~mm}$ ID) fluoropolymer capillary, conductivity detector and fraction collection valve. Sample volumes up 150 microliters could be injected, focused and collected. In order to achieve a high recovery and enrichment, factors including electric current, sample amount and matrix were investigated experimentally as well as by computer simulation.

The second, laboratory constructed, system was designed in a flat arrangement where sample zones migrated towards a fraction collection well. This allowed focusing of $15 \mathrm{ml}$ sample volumes in a $110 \mathrm{~mm}$ device in less than 1 hour. Position of the migrating zone was monitored by laser-induced fluorescence. While a discontinuous electrolyte system was used, the selected geometry did not lead to a typical isotachophoretic migration when operated at constant current, constant voltage or constant power modes. These experimental findings were confirmed by theoretical descriptions derived for each operation mode. Samples dissolved in the terminating electrolyte or in saline solutions were processed in both systems. The DNA content in the collected fractions were further analyzed by fluorescence spectrometry and chip capillary electrophoresis.

In conclusion, we have developed two simple, preparative methods for DNA concentration and purification. The first method, based on a capillary ITP, can process sample volumes up to 150 microliters. The second method, based on the flat channel design, allows processing of $15 \mathrm{ml}$ sample volumes. This is by far the largest described focusing capacity. In the discontinuous electrolyte system, the sample enrichment factor is limited only by the system geometry and even higher loading capacities are possible if needed.

\section{Rare Variant of FAP-GAPPS Syndrome with Severe Risk of Gastric Polyposis and Adenocarcinoma with a Need for Gastrectomy - A High Frequency in the Czech Republic}

L. Foretova, M. Navratilova, M. Svoboda, J. Hazova, P. Vasickova, E. Stahlova-Hrabincova, E. Machackova

Department of Cancer Epidemiology and Genetics, Masaryk Memorial Cancer Institute, Brno, Czech Republic

Gastric adenocarcinoma and proximal polyposis of the stomach (GAPPS) is a rare variant of familial adenomatous polyposis (FAP). It is an autosomal-dominant cancer-predisposition syndrome with a massive polyposis of stomach and a significant risk of gastric adenocarcinoma. Li et al., 2016, described 4 point mutations in the YY1 binding site of the APC gene 1B promoter associated with GAPPS syndrome.

Sanger Sequencing of APC promoter 1B was performed in 34 Czech families with polyposis of stomach, colon or gastric adenocarcinoma. In seven families with gastric polyposis or gastric adenocarcinoma, pathogenic variant in $A P C 1 \mathrm{~B}$ promoter NM_001127511: c.-191T $>C$ associated with GAPPS was detected. No mutation in promoter $1 \mathrm{~B}$ of APC gene was detected in patients with colonic polyposis with no mutation previously found in APC gene.

In 17 positively tested individuals from 7 families, 15 had massive gastric polyposis with more than 100 fundic gland polyps, 3 of them already died of adenocarcinoma, one proband 
had metastatic gastric tubular adenocarcinoma at 28 , and died at 29; one is treated now with adenocarcinoma at 42 . No polyposis was detected yet by gastroscopy in a carrier woman born at 1987 and in another carrier, gastroscopy was not done yet. Prophylactic gastrectomy was already recommended to all carriers with detected massive polyposis. In five of them it was already done, in a carrier born 1988, unfortunately G2 gastric adenocarcinoma was already present. Other prophylactic surgeries are planned.

Point mutation c. $-191 \mathrm{~T}>\mathrm{C}$ in $1 \mathrm{~B}$ promoter of $A P C$ gene is a frequent cause of aggressive gastric polyposis in the Czech Republic, with a high risk of gastric adenocarcinoma, even in a young age. Positively tested individuals are recommended to high risk oncology clinic. Information about the possible prevention by gastrectomy is necessary.

This syndrome was not known until recently and gastrectomies in gastric polyposis were not frequently recommended. Genetic testing reveals hereditary cause of the disease, enables predictive testing of all relatives, polyposis can be discovered early and gastrectomy planned. There is no other efficient prevention of gastric cancer in GAPPS. In the future, any research for other than surgical prevention of massive gastric polyposis will be needed.

Supported by Czech Ministry of Health: MH CZ - DRO (MMCI, 00209805).

\section{Next-Generation Sequencing to Identify Rare Variants Related to FL-Toxicity \\ F. Ecca ${ }^{\mathrm{a}, \mathrm{b}}$, E. De Mattia ${ }^{\mathrm{a}}$, F. Serra ${ }^{\mathrm{c}}$, R. Roncato ${ }^{\mathrm{a}}$, E. Dreussi ${ }^{\mathrm{a}}$, L. Romanato a , A. Buonadonna ', A. De Paolie, M. Berretta ${ }^{\text {d }}$, E. Minif, S. Nobilis, G. Toffoli ${ }^{\mathrm{a}}$, E. Cecchin ${ }^{\mathrm{a}}$ \\ aExperimental and Clinical Pharmacology, Centro Di Riferimento Oncologico - National Cancer Institute, Aviano, Italy, ${ }^{\mathrm{b}} \mathrm{PhD}$ School, University of Udine, Italy; ${ }^{\mathrm{C} C}$ Control of Gene Expression INSERM U 1065, C3M, Equipe10, University of Nice Sophia- Antipolis 151, route de St-Antoine-de-Ginestière B.P. 23194 06204 - Nice CEDEX 3 France; ${ }^{d}$ Medical Oncology Department, Centro Di Riferimento Oncologico - National Cancer Institute, Aviano, Italy; ${ }^{~}$ Radiation Oncology Department, Centro Di Riferimento Oncologico - National Cancer Institute, Aviano, Italy; fSection of Internal Medicine, Department of Experimental and Clinical Medicine, University of Florence, Italy; ${ }^{9}$ Section of Clinical Pharmacology and Oncology, Department of Health Sciences, University of Florence, Italy}

Fluoropyrimidines (FL) are frequently used in several solid tumours treatment. Unfortunately, about $26 \%$ of patient treated with FL will develop unpredictable severe to life-threatening toxicity (grade $\geq 3$ ). Common SNPs provide partial explanation to the overall adverse reactions. However, most variants affecting proteins phenotype in humans, including those responsible for drugs ADME, were reported to be novel and rare (Lek et al, 2016; Kozyra et al, 2017).

We aimed to map 54 pharmacogenes involved in the FL ADME and folate pathway to identify novel markers of severe FL-related toxicity in a selected group of patients with severe FL-related toxicity phenotype.

Submitted Abstracts from Summer School Participants
For the present project, 109 solid cancer patients treated with a FL-based regimen, with an available blood specimen, were retrospectively selected, for undergoing germline NGS analysis, based on grade $\geq 3$ toxicity occurrence and absence of acknowledged DPYD risk variants (DPYD*2A, DPYD*13, DPYD-c2846, and DPYD-HapB3). PharmGKB resources and literature data were used for genes selection in different FL-related pathways such as: metabolism and transport, folate pathway and regulation of expression of DPYD and other pharmacogenes.

A custom NimbleGenSeqCap EZ Choice (Roche, Inc.) which covers the CDS and UTRs of 54 target genes, including the flanked splice junction regions was designed. An additional region of $3 \mathrm{kbp}$ was included to address the promoter genetic variants for 3 genes (DPYD, MTHFR and TYMS). The libraries were sent for sequencing to generate $2 \times 150$ bp paired-end reads using a MiSeq instrument (Illumina, Inc.).

We have currently analysed 72/109 patients. A preliminary analysis was focused on $D P Y D$ sequencing results, playing a pivotal role in FL ADME. We evaluated the number of total detected variants and then we filtered the results to highlight rare variants (MAF1\%). For those variants different in silico tools were applied to point out potential functional effect on the protein.

By a preliminary analysis, five novel variants in the DPYD gene were identified. Four of them were missense exonic and one was located in the 3'UTR. Novel variants were analytically validated by direct Sanger sequencing. In addition, one previously reported rare exonic missense variant was detected in one patient.

In conclusion we preliminarily demonstrated that rare and novel genetic variants in DPYD can be detected in patients with an extreme toxicity phenotype and can potentially account for a defective FL-detoxification. We plan to complete the sequencing of all patients and we will try to demonstrate the functional role of these variants through functional assays.

\section{Trp53 Signaling in Chemically Induced Lung Adenocarcinoma \\ M. Oplopoiou, D. Kati, G. Ntaliarda, I. Giopanou, \\ V. Papaleonidopoulos, I. Lilis, G.T. Stathopoulos}

Laboratory for Molecular Respiratory Carcinogenesis, Department of Physiology, Faculty of Medicine, University of Patras, Rio, Achaia, 26504, Greece; and Comprehensive Pneumology Center, Helmholtz Zentrum München, Member of the German Center for Lung Research (DZL), Munich, Bavaria, 81377, Germany

Background: Lung adenocarcinoma is the number one cancer killer in the world and a public health burden, accounting for almost $50 \%$ of non-small-cell lung cancers and $40 \%$ of lung tumors overall. Activating mutations in the KRAS oncogene together with inactivating mutations in the tumor suppressor TP53 are key drivers of lung adenocarcinoma and are also present in urethaneinduced lung tumors of mice. However, little is known about the timing and the function of TP53 mutations.

Aim: To characterize the induction of Trp53 mutations in mice by chemical constituents of tobacco smoke and to identify their function. 
Methods: Mice received repetitive intraperitoneal urethane and were examined after six months for lung tumors. Multiple cell lines were isolated from lung tumors and were analyzed for Trp53 loss. Trp53f/f mice received intratracheal adenovirus encoding Cre recombinase two weeks before or after urethane treatment, and tumors were examined after six months. Trp53f/wt kaı Trp53f/f mice were intercrossed with Lyz2.Cre, Sftpc.Cre and Scgbla1.CreFVB mice and double heterozygote offspring was treated with urethane.

Results: $35,7 \%$ of urethane-induced tumors and six of nine cell lines examined featured heterozygous or homozygous Trp53 loss. In addition to primary tumors, cell lines derived thereof featured marked heterogeneity in Trp53 loss patterns, even within the same lung. Adenovirus-mediated Trp53 deletion had no effect on subcutaneous lung adenocarcinoma growth nor on de novo urethaneinduced tumorigenesis, regardless of the timing of Trp53 deletion. However, specifically Trp53f/wt;Lyz2.Cre and Trp53f/f;Sftpc.Cre mice had increased numbers and diameters of lung tumors induced by urethane respectively, compared with all other lineagerestricted Trp53-deleted mice. Additional, a cancer cell line with Trp53 loss and one without were identified for possible abnormalities at their mitotic spindle and therefore the role of that gene at cell division and woundhealing assay and SC injection were followed to check EMT phenomenon and metastasis.

Conclusions: $\operatorname{Trp} 53$ loss is caused by the tobacco carcinogen urethane at the tumor initiation-early promotion phase, and functions to promote not only tumor establishment, but also growth. Trp53 tumor promoting functions are restricted to Lyz2 and Sftpc expressing alveolar type II cells with stemness potential. Trp53 loss also plays important role at cell invasion and metastasis.

\section{Overview of Cost-Utility Analysis and Reimbursement of Individualized Medicine Interventions}

S. Simeonidis ${ }^{\mathrm{a}}$, A. Vozikis ${ }^{\mathrm{b}}$, G.P. Patrinos ${ }^{\mathrm{a}}$

aUniversity of Patras School of Health Sciences, Department of Pharmacy, Patras, Greece; bUniversity of Piraeus, Economics Department, Piraeus, Greece

Introducing pharmacogenomic tests in clinical practice leads to the design of new, individualized interventions with as much efficacy and safety as possible in order to improve the quality of patients' life. Unlike the ever-increasing social needs, funding for the public health sector is constantly decreasing; the new interventions that have emerged should be evaluated in terms of both their effectiveness and their overall costs.

A systematic review of 96 economic evaluation studies (articles) was conducted with the aim of presenting the basic principles of applied health policies, as far as economic evaluation and reimbursement of individualized interventions are concerned. 39 studies were conducted in the USA and 36 studies were conducted in a European country.

The results indicate that 40 studies were a combination of costeffectiveness and cost-utility analyses, 27 studies only included cost-utility analyses, and 17 studies only included cost-effectiveness analyses. Interestingly, most of the studies $(n=80)$ were based on hypothetical cohorts. Only 3 cost minimization analyses were identified. Furthermore, in 26 studies the outcome of the interventions was estimated in quality-adjusted life years (QALYs), in 38 studies the outcome was estimated in both "physical units", including life years (LYs), and QALYs, while only in 9 studies the outcome was estimated in LYs. It is noteworthy that in 76 studies the cost of the interventions was estimated only in terms of the direct medical cost, thus the studies were conducted mostly by the health payers' perspective.

Diagnostic interventions for breast cancer that contained the MammaPrint ${ }^{\circledR}$ test and therapeutic interventions that contained pharmacogenomic tests for the UGT1A1 and CYP2C19 genes for colorectal cancer and acute coronary syndrome respectively, proved to be "dominant". These interventions were more effective and less costly than the "old" interventions. Indicatively, diagnostic interventions for Lynch syndrome and papillary thyroid cancer that contained pharmacogenomic tests for $M M R$ and $B R A F$ genes, respectively, had incremental cost-utility ratios (ICURs) of \$26000/QALY and \$33,96/QALY. Therapeutic interventions that contained pharmacogenomic tests for VKORC1/CYP2C9, HLA$B^{*}$ 15:02 and TPMT genes for atrial fibrillation, epilepsy and idiopathic pulmonary fibrosis, respectively, had ICURs of $<\$ 50000 /$ QALY, \$29750/QALY and \$49156/QALY.

In addition, diagnostic interventions for breast cancer that contained the Oncotype $\mathrm{DX}^{\circledR}$ or genomic tests for the BRCA1/2 genes had ICURs of $£ 5529 / \mathrm{Q} A L Y$ and $£ 2079$ - $£ 4339 / \mathrm{Q} A L Y$. Therapeutic interventions that contained pharmacogenomic tests for VKORC1/CYP2C9 and HLA-A*31:01 genes for atrial fibrillation and epilepsy, respectively, had ICURs of $£ 6702-£ 20671 / Q A L Y$ and $£ 12808 /$ QALY.

Furthermore, genomic/pharmacogenomic tests for $B R C A 1 / 2$, UGT1A1, EGFR, KRAS, BRAF, ALK, HLA-A, HLA-B, HER-2, TPMT and MMR genes, as well as Oncotype $\mathrm{DX}^{\circledR}$, were found to be reimbursed by Medicare.

Briefly, the reimbursement criteria of pharmacogenomic tests are: (a) The willingness to pay threshold, (b) clinical utility and benefit of the pharmacogenomic tests, (c) FDA recommendations for genetic analysis before medicine administration and (d) characteristics of patients (high-risk groups, family history of illness).

In conclusion, the high cost of individualized interventions is the main inhibitor of non-adoption and non-reimbursement of pharmacogenomic tests. The economic evaluation of new interventions ensures the proper allocation of resources in order to provide the best benefit to patients and to maintain the sustainability of national healthcare systems. However, the analysis of cost and effectiveness should be done by a more societal rather than strictly payers' perspective.
2018 Golden Helix Summer School Rare Genomics 


\section{Identification of Two Novel Candidate Loci to Predict Response Effectiveness to Lithium Treatment in Sardinian Patients with Bipolar Disorder}

E.E. Tsermpini ${ }^{\mathrm{a}}$, K. Mitropoulos ${ }^{\mathrm{b}}$, E. Zafeiri ${ }^{\mathrm{a}}$, A. Kanterakis, ${ }^{\mathrm{a}, \mathrm{c}}$, A. Squassina ${ }^{\mathrm{d}}$, D. Congiu ${ }^{\mathrm{d}}$, G. Severino $^{\mathrm{d}}$, M. Del Zompo ${ }^{\mathrm{d}, \mathrm{e}}$, G.P. Patrinos ${ }^{\mathrm{a}}$

aUniversity of Patras, School of Health Sciences, Department of Pharmacy, Patras, Greece, bUniversity of Athens, School of Medicine, Athens, Greece, 'Institute of Computer Science, Foundation for Research and Technology, Heraklion, Crete, Greece; dUniversity of Cagliari, Department of Neuroscience, Section of Clinical Pharmacology, Monserrato, Cagliari, Italy, eUniversity Hospital of Cagliari, Unit of Clinical Pharmacology, Cagliari, Italy

Background: Bipolar Disorder (BD) is a serious psychiatric illness characterized by manic and depressive episodes affecting $1-5 \%$ of the general population. Among the various mood-stabilizing treatments, lithium represents one of the main treatment modalities in the therapeutic management of BD. However, apart from the relatively high rate of responders to lithium treatment, a significant percentage of patients fail to fully respond to lithium treatment, presenting with patterns of partial or non-response. This variability might be influenced by genetic factors, even though findings have been so far inconclusive. We have previously shown that the $A C C N 1$ gene is a potential candidate locus for response to lithium treatment that would serve as a genomic biomarker of lithium efficacy for BD patients (Squassina et al., Pharmacogenomics 2011;12(11):1559-1169).

Materials and Methods: Phenotypic assessment of lithium response was made using the retrospective criteria of long-term treatment response scale. We have genotyped $52 \mathrm{BD}$ patients evenly distributed at the extreme ends of the treatment response scale using Affymetrix 6.0 single nucleotide polymorphism (SNP) arrays. Data were subsequently analyzed using different analysis tools, used in our previous work, namely EIGENSTRAT filtering and KINGSHIP analysis.

Results: Our results confirmed our previous findings (Squassina et al., 2011) and further revealed two additional candidate loci, associated with lithium treatment response $\left(\mathrm{p}=4.113 \times 10^{-5}\right.$ $-5.324 \times 10^{-7}$ ). In particular, application of EIGENSTRAT (PCA) filtering identified two SNPs located in the ABCG2 gene, as well as one SNP in the TGFB1 gene to be possibly associated with lithium response.

Conclusions: These association signals are being selected for validation and currently being subjected to extended genotyping in the whole sample of BD patients characterized for lithium response to confirm this hypothesis.

\section{ZBTB7A Conservation in Primates Establishes its Significance as an Epigenetic Treatment Predictor for $\boldsymbol{\beta}$-Hemoglobinopathies}

V. Chondrou', G.P. Patrinos ${ }^{\mathrm{b}}$, A. Papachatzopoulou' ${ }^{\mathrm{c}}$, A. Sgourou ${ }^{\mathrm{a}}$

aHellenic Open University, School of Science and Technology, Biology Laboratory, buniversity of Patras, School of Health Sciences, Department of Pharmacy, Laboratory of Pharmacogenomics and Individualized Therapy, 'University of

Patras, Medical Faculty, Laboratory of General Biology, Greece

The conserved sequences across species have always provided possible avenues to understand the complete picture of human gene's entity, significance and cell operation by their convergent evolution. Comparative genomics support the view that across critical transcription-factor binding sites, sites that contribute more to the total regulatory activity accumulate fewer substitutions.

ZBTB7A human gene encodes the zinc finger and BTB domaincontaining protein 7A, LRF. ZBTB7A/LRF binds to CG-rich regions and recruits histone deacetylases to gene promoters leading to a closed chromatin conformation that promotes gene silencing. In humans, the transcription factor ZBTB7A/LRF is necessary for hematopoietic stem cell maintenance and has been found to repress the fetal hemoglobin ( $\mathrm{HbF}$ ) independently from BCL11A, during fetal to adult hemoglobin switching, via an epigenetic mechanism. In mice, loss of LRF leads to increased apoptosis of erythroid precursors and lethal anemia, while the LRF knockdown increases $\mathrm{HbF}$ expression but delays human erythroid differentiation.

The ZBTB/POK proteins are an evolutionarily conserved family of transcription factors. They are characterized by an N-terminal BTB domain (broad-complex, tram-track, and bric-a-brac) and one or more C-terminal C2H2/Krüppel-type zinc finger (ZF) domains. The BTB domain mediates the homo or hetero-dimerization between ZBTB proteins and interacts with transcriptional co-factors and histone modification proteins (SIN3A, BCOR, NCOR-1/2, HDAC) altering the conformation of chromatin. ZF domains bind to DNA regulatory regions of targeted genes by a sequence specific manner ensuring transcriptional specificity.

The human ZBTB7A sequence aligned to its orthologues showed $70-100 \%$ similarity in primates, $\sim 60 \%$ in sauropsida and $65-90 \%$ in rodents and related species etc (ensembl.org/), indicating a highly sustained conservation on the DNA level. Furthermore, data from RNA-seq expression profile across 16 selected tissues (NHPRTR project) revealed that humans exhibit higher expression levels compared with old world primates and new world primates.

The fact that ZBTB7A/LRF putative genes homologs' comparisons and their conserved protein domain architectures showed a range from high to exact match among species (using the batch$\mathrm{CD}$, conserved domain, search interface from NCBI and UniProt data bases), indicates its essential role during evolution. The BTB domain exhibits in all primates including humans, preferential interactions with chromatin-associated factors. These findings in combination with its repressing role on $\mathrm{HbF}$ during human erythropoiesis after birth as well as its different methylation pattern in response to hydroxyurea treatment (Chondrou et al, unpublished data) hold promise for the ZBTB7A/LRF establishment as an emerging epigenetic biomarker for the $\beta$-hemoglobinopathies. 


\section{Diagnostic Agrigenomics: Surveying the Brewing Reservoir of Emerging and Rare Infections}

A. Stathoulias ${ }^{\mathrm{a}}$, E. Barba ${ }^{\mathrm{a}}$, Y. Manousopoulos ${ }^{\mathrm{b}}$, A. Velegraki $^{\mathrm{c}}$, M.E. Kambouris ${ }^{\mathrm{a}}$, G.P. Patrinos ${ }^{\mathrm{a}}$

aUniversity of Patras School of Health Sciences, Department of Pharmacy, Patras, Greece, bPlant Protection Division of Patras, Institute of Industrial and Forage Plants, Patras, Greece, 'Departments of Microbiology, School of Medicine, National and Kapodistrian University of Athens, Athens, Greece

The community environments house complex microbiomes, mostly saprobiotic but also parasitic to quite an extend. Parasitism usually features steady and narrow host selection, but more generic trends arise, especially with opportunistic pathogens which usually double as saprobiotes.

Genomic homogeneity of current crops facilitates transmission and extreme propagation of adapted pathogens, creating ever higher loads. On the other end, a host of low immunity, be that due to occasional (pregnancy, fatigue, seasonal or random disease) or systematic events (cancer, transplantation, HIV infection) appears at the ready to provide new hosts, susceptible although highly different than the original pant and or wildlife. Both the massive transportation of raw produce directly to marketing areas and the few immunodeficiency events in the most exposed, rural populations create interfaces for new microbiotes, especially fungi, to cross the host-species barrier and colonize and then adapt to human hosts to become opportunistic pathogens-a probabilistic event becoming more probable with the increase and propagation of any single environmental contaminant. The mechanism is responsible for the emerging and rare infections, especially of fungal causes.

Thus, given that most pathogens ingressed into the human host from another source or reservoir (hostile interface), Public Health extends to agrisector and environment to survey tendencies and near-future infectious events. Molecular detection diagnosis by medical protocols, as generic as possible or transferable with the least adaptation from human diagnostics, can be used for plant protection purposes providing an early warning element for Public Health. Applicable techniques include the New Generation Sequencing, to dissect the microbial populations by both registering and identifying constituting microbiota from plant, animal and soil samples, but specific PCR followed by RFLP is highly targetable targeted and applicable in dispersed facilities so as to identify pathogens by species. 